\title{
Self-generated and Externally Driven Current Oscillations in n-GaAs
}

\author{
J. Spangler and W. Prettl \\ Institut für Angewandte Physik, Universität Regensburg, 93040 Regensburg, Germany
}

Received April 26, 1994; accepted July 8, 1994

\begin{abstract}
Experimental investigations of self-generated and externally driven nonlinear current oscillations due to impact ionization of shallow impurities in $\mathrm{n}$-type GaAs at low temperatures are presented. The regular relaxation oscillations which appear at the onset of breakdown are destabilized by a magnetic field normal to the epitaxial layer and multifrequency oscillations and chaotic fluctuations following the Ruelle-Takens-Newhouse scenario can be found. Driving the self-generated oscillations with an external periodic bias voltage quasiperiodicity, mode locking, interaction and overlap of mode locked resonances and period doubling can be observed depending on frequency and amplitude of the external force. The reconstructed return maps, recorded phase diagrams and Devil's staircase agree with theoretical predictions based on the circle map and give new evidence of the universality of the transition from quasiperiodicity to chaos.
\end{abstract}

\section{Introduction}

Transitions into chaos due to two competing frequencies have been observed in several nonlinear dissipative dynamical systems [1-11]. In all cases the oscillation modes were self-generated or induced by an external periodical force. Theoretical investigations of this quasiperiodic route to chaos are based on the circle map and had led to quantitative predictions of universal scaling behaviour [12-18]. These predictions have been verified in experiments on hydrodynamic [1-3], electrical [4-6], and solid state systems [7-11].

In semiconductors nonlinear current-voltage characteristics, negative differential conductivity, instabilities like selfsustained oscillations, bifurcations and the spontaneous formation of current filaments are manifestations of the nonlinear dynamical properties of the charge carrier transport. In particular high-purity semiconductors show strongly nonlinear current-voltage characteristics at temperatures where the thermal energy is less than the binding energy of residual shallow impurities.

The nonlinearities are caused by impact ionization of shallow impurities which represents the dominant autocatalytic free carrier generation process in extrinsic semiconductors. At a critical electric field strength an avalanche breakdown occurs, similar to the electric breakdown in gases. Free carriers gain sufficient energy so that the impact ionization rate of shallow impurities exceeds the recombination rate for low carrier concentrations. This leads to a rapid increase of the current at a practically constant voltage indicating a filamentary current flow. At the avalanche breakdown a strongly ionized thin channel is formed in the sample carrying the total current. The growth of current at constant voltage is then caused by a proportional increase of the ionized volume. In many cases the transition from high-ohmic pre-breakdown behavior to filamentary current flow is associated with autonomous oscillations.

The observed current oscillations were analyzed in terms of nonlinear dynamics and different routes to chaos could be found [19-24]. Progress on understanding of these phenomena on a microscopic basis has been obtained in the framework of semiclassical transport theory applying rate equation models for the kinetics of the impurities [25].

In this paper we summarize briefly the properties of selfgenerated oscillations in $\mathrm{n}-\mathrm{GaAs}$ epitaxial layers. Attention will be drawn on the importance of the spatial degrees of freedom of the filament borders and an external magnetic field for the occurrence of multifrequency oscillations and chaotic fluctuations. Then experimental results of the coupling of internal oscillation to a periodic drive will be presented. Self-sustained oscillations were subjected to a periodic voltage added to a constant bias offset. The response of the sample to the sinusoidal voltage has been measured as function of amplitude and frequency of the external force. The results are analyzed in terms of the circle map theory. In a control parameter range of regular relaxation oscillations a quasiperiodic transition to chaos has been found. These investigations give new experimental evidence of the universality of the quasiperiodic transition to chaos. Both self-sustained oscillations as well as coupling of an external periodic driving force to internal oscillations are of interest to establish the underlying microscopic physical mechanisms.

\section{Experimental}

The measurements were carried out on a $16 \mu \mathrm{m}$ thick $\mathrm{n}$-GaAs epitaxial layer of $n=1.3 \times 10^{14} \mathrm{~cm}^{-3}$ electron concentration and $\mu=8.9 \times 10^{4} \mathrm{~cm}^{2} /$ Vs mobility at $77 \mathrm{~K}$ corresponding to $N_{D}=5.7 \times 10^{14} \mathrm{~cm}^{-3}$ donor density at a compensation ratio of $77 \%$. The layer was grown on a semiinsulating GaAs substrate of $3 \times 3 \mathrm{~mm}^{2}$ area. At the center of two opposite edges of the sample ohmic contacts were prepared by alloying Indium spheres of $0.5 \mathrm{~mm}$ diameter. The free distance between these two point contacts was $2 \mathrm{~mm}$. The sample was fixed in the center of a superconducting magnet and immersed in liquid helium at $4.2 \mathrm{~K}$. A mount was used which allowed a precise orientation of the sample in the magnetic field. Thermal background radiation was suppressed by a cold metallic shield. The sample was biased in series with $100 \mathrm{k} \Omega$ load resistor. The voltage drop across the sample was measured as a function of time with a waveform digitizer. The recorded time series were transferred to a computer for further numercial evaluation. 


\section{Self-generated current oscillations}

In $\mathrm{n}$-GaAs without a magnetic field only regular relaxation oscillations have been observed which are strictly periodic. The frequency of the oscillations rises with increasing bias voltage. As a function of the average bias current the oscillation frequency grows almost linearly from $0.7 \mathrm{MHz}$ at the threshold of the instability to $3.5 \mathrm{MHz}$ when a stable current filament is abruptly formed. This behavior of the regular relaxation oscillations is caused by a repetitive ignition and extinction of a current filament and the frequency is determined by the charging current of the parasitic capacitance in the sample circuit.

Small magnetic fields of the order of $100 \mathrm{mT}$ have a pronounced effect on the temporal structure of the oscillations (Fig. 1). The appearance of complex oscillations, however, crucially depends on the orientation of the magnetic field vector with respect to the current and the plane of the epitaxial layer. When the magnetic field is parallel to the current [Fig. 1(a)] only regular oscillations and stable current flow have been observed as in the case of $B=0$. This is the case at least up to $2 \mathrm{~T}$, the highest magnetic field that has been applied. Increasing the magnetic field reduces the current where the transition from relaxation oscillations to a stationary current filament takes place. As a result a longitudinal magnetic field stabilizes the filamentary current flow [26] and suppresses oscillations.

Measurements with the magnetic field in the plane of the epitaxial layer and perpendicular to the current are shown in configuration Fig. 1(b). In this case again only periodic relaxation oscillations have been found for magnetic field strengths up to about $500 \mathrm{mT}$. At higher magnetic fields complex oscillations occur which have not yet been well characterized. This oscillations are most probably due to the dynamical Hall effect proposed by Hüpper and Schöll [27]. Here the Lorentz force and the Hall field are normal to the plane of the semiconductor. The dynamical Hall effect yields purely temporal oscillations and chaotic fluctuations of the free carrier density without involving spatial degrees of freedom.

In the third configuration investigated [Fig. 1(c)] the magnetic field is normal to the epitaxial layer and perpen-

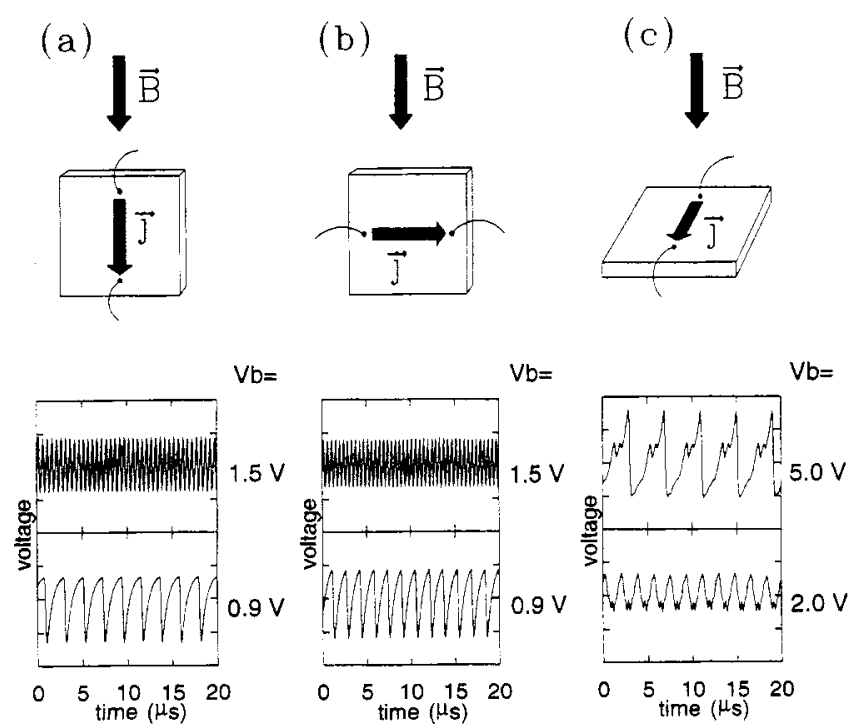

Fig. 1. Geometrical configurations of the sample surface, the current $j$ and the magnetic field $B$ and the resulting oscillations forms at $B=100 \mathrm{mT}$. dicular to the current. Now complex oscillations can be observed depending on the magnetic field and the applied bias voltage. Typically Ruelle-Takens-Newhouse scenarios and intermittency could be recognized. The significant difference to the two previous configurations is that in this case a Hall electric field arises in the plane of the semiconductor layer.

This results demonstrate the importance of the Lorentz force and the spatial degrees of freedom of the filament for the occurrence of multifrequency oscillations and chaos in the filamentary current flow regime of thin n-GaAs layers. A magnetic field normal to the current flow accumulates space charges by the Hall effect in the boundaries of the current filament because of the high mobility of the hot electrons in the filament. The filament borders in the plane of the thin film are movable and represent spatial degrees of freedom of the filament dynamics. If these borders are charged due to a magnetic field pointing normal to the semiconductor place different modes of cyclic generation-recombination processes occur in the filament and at the opposite edges of the filament. Coupling of these modes by free carrier drift and diffusion yields quasiperiodic and frequency locked oscillations as well as chaos. If the magnetic field is normal to the current but in the plane of the semiconductor charge accumulation is on top and bottom of the layer because the filament fills the thickness of the film. Boundary oscillations are suppressed due to this confinement of free carriers and increased free carrier recombination at the interfaces of the thin semiconductor layer. Localized fluctuations of carriers may still occur as shown with the theory of the dynamic Hall effect. If the magnetic field is parallel to the current filament no Lorentz force arises and, consequently, the filamentary current flow stays stationary.

The complex current oscillations for a magnetic field of $100 \mathrm{mT}$ transverse to the current and normal to the sample surface are presented in more detail. Time series have been measured for different voltages across the sample in series with the load resistor. These data have been used to calculate power spectra, phase space reconstructions and Poincare sections. Also the fractal dimension $d$ of the attractor were evaluated using the method of Grassberger and Procaccia [28]. The results are shown in Fig. 2. For a voltage of $1.500 \mathrm{~V}$ [Fig. 2(a)] stationary current flow below breakdown

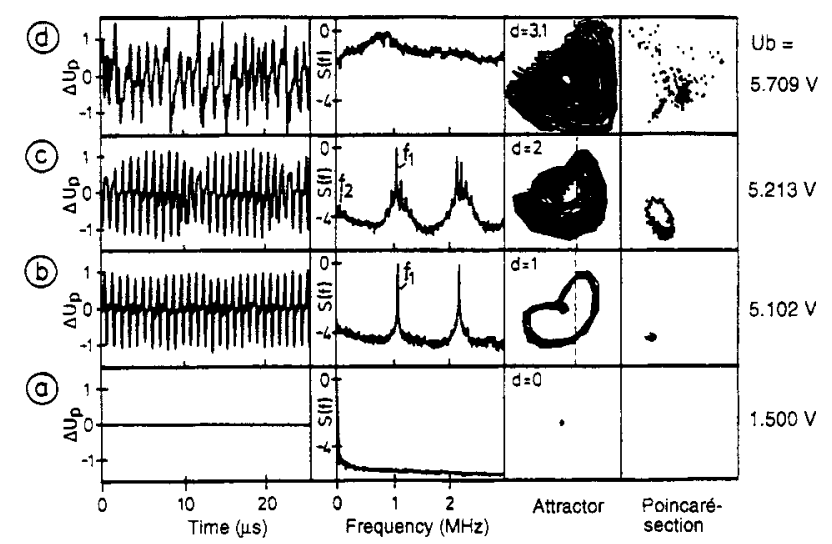

Fig. 2. Ruelle-Takens-Newhouse scenario for a transversal magnetic field $B=100 \mathrm{mT}$. (a) Stationary current flow breakdown; (b) regular oscillations with fundamental frequency $f_{1}$; (c) quasiperiodic oscillations with $f_{1}$ and $f_{2}$; (d) chaotic fluctuation with fractal dimension of 3.1. 


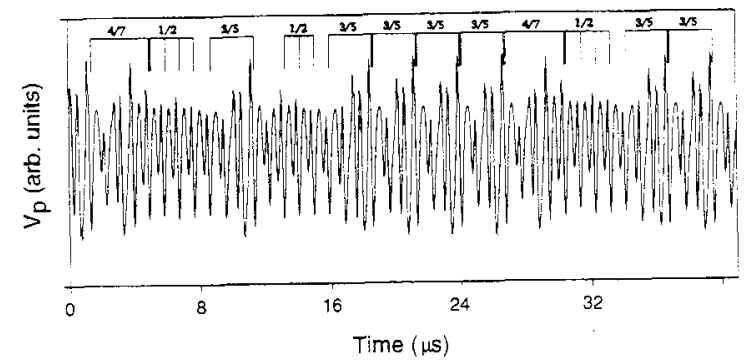

Fig. 3. Time series for $f_{d}=1.097 \mathrm{MHz}$ and $V_{d}=0.82 \mathrm{~V}$ above the critical line. Overlapping and mixing of resonances in an erratic way with frequency ratios $1 / 2,3 / 5$ and $4 / 7$.

has been observed. Raising the voltage a sequence of three Hopf bifurcations occurs which allows to recognize a Ruelle-Takens-Newhouse scenario [29]. At 5.102 V [Fig. 2(b)] a regular relaxation oscillation with one fundamental frequency $f_{1}$ arise which proceed into quasiperiodic oscillations with the two frequencies $f_{1}$ and $f_{2}$ at $5.213 \mathrm{~V}$ [Fig. 2(c)]. The phase portrait and the Poincare section clearly show a torus. Finally in Fig. 2(c) at $5.709 \mathrm{~V}$ chaotic fluctuations are displayed with a fractal dimension of 3.1 .

\section{Externally driven current oscillations}

In order to investigate externally driven oscillations as a function of frequency and amplitude of the driving voltage the series circuit of the sample and the load resistor was biased with a frequency synthesizer. For all measurements the same offset voltage was used and all measurements were carried out at zero field because in this case the relaxation oscillations are very stable and have low noise.

For the presentation of the universal scaling properties we will follow the circle map model [16]. This one dimen-
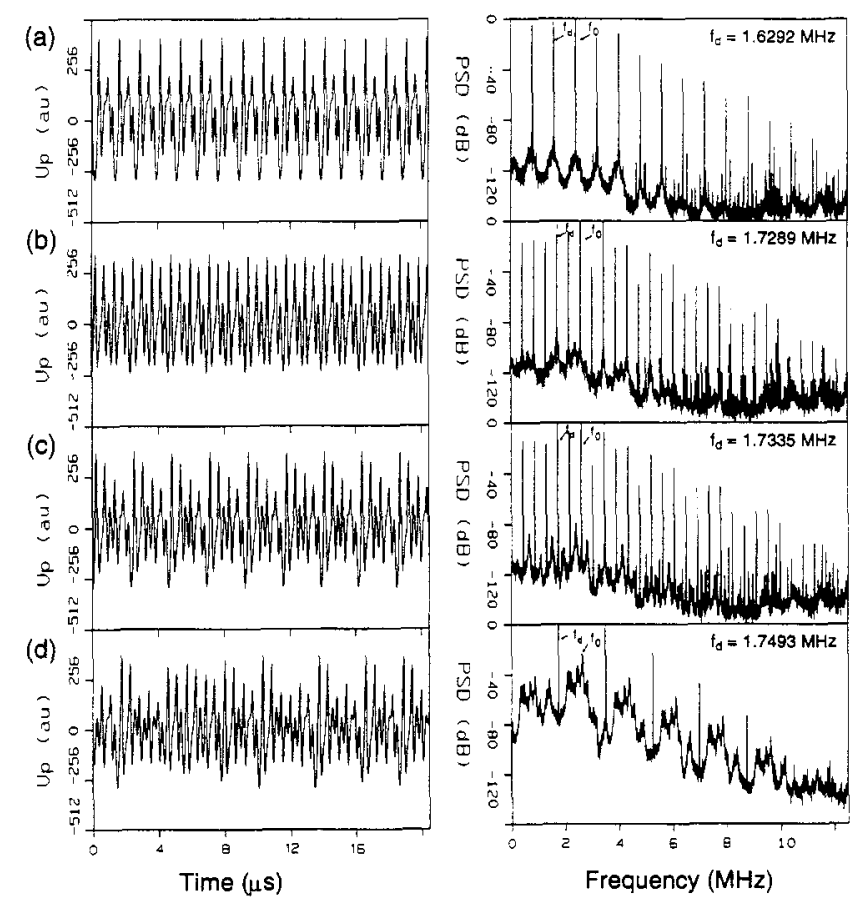

Fig. 4. Time series and power spectra for four different driving frequencies $f_{d}$ and a driving amplitude $V_{d}=1.0 \mathrm{~V}$ above the critical line. Transition into chaos by three subsequent period doublings after Feigenbaum. sional map of the circle onto itself

$\Theta_{n+1}=f_{\Omega}\left(\Theta_{n}\right)=\left(\Theta_{n}+\Omega-\frac{K}{2 \pi} \sin 2 \pi \Theta_{n}\right) \bmod 1$

can describe quasiperiodicity, mode locking and the transition to chaos of two competing frequencies. The relevant control parameters are the ratio $\Omega=f_{d} / f_{0}=P / Q$ between the drive frequency $f_{d}$ and the frequency $f_{0}$ of the selfgenerated oscillation and the amplitude $K$ of the sinusoidal nonlinearity, which is related in our experiment to the amplitude $V_{d}$ of the external drive.

Choosing a driving amplitude below the critical line, quasiperiodicity and mode locking can be observed. This could be measured in the orbits and Poincare sections for different parameter sets below the critical line. In the Poincaré section the quasiperiodic motion manifests itself as a circle and the mode locked motion in points whose number is equal to the denominator $Q$ of the frequency ratio $P / Q$.

If the driving amplitude is above the critical line $K=1$ the circle map looses invertibility and chaotic behavior arises because the orbit jumps between the various overlapping resonances in an erratic way and a transition to chaos takes place. This overlapping can clearly be seen in a time series in Fig. 3 where lockings with frequency ratio 1/2, 3/5 and $4 / 7$ mix. Also a breakup of the invariant two-torus occurs which manifests itself by the appearance of wrinkles in the Poincaré section.

For $K>1$ the map also develops quadratic maxima and minima. Iterations of this type of mapping exhibit infinite series of period doubling leading into chaos characterized as Feigenbaum scenario [30]. As depicted in Fig. 4 by time series and power spectra three period doubling cascades could be resolved before chaotic fluctuations set in.

\section{Return maps}

For the reconstruction of return maps orbits were determined from time series by the method of delay coordinates. Then the Poincaré section was divided into sectors and the map was constructed from the radian which was normalized to 1 . This method was chosen because the Poincaré section deviates from a circle. The smearing of the observed data set is mostly due to noise and drift in the experimental system. In the absence of these the points should fall on a single curve.

In Fig. $5(\mathrm{a})-(\mathrm{c})$ in the first sequence for $0.14 \mathrm{~V}$ the driving voltage was chosen well below the critical line. Here almost only quasiperiodic oscillations exist. The almost constant gradient in the map is reflected by the homogeneous distribution of the intersections and a constant broadening of the circle line by noise in the Poincare section. For $0.63 \mathrm{~V}$ narrow below the critical value in Fig. 5(c)-(e) a decrease of the gradient at $x_{n}=0$ in the return map can be seen. Furthermore the inhomogeneous distribution of the intersection points in the Poincare section and the nonergodic course of the space trajectory states the general tendency for mode locking. Finally orbit, Poincare section and return map for a critical trajectory are plotted in Fig. 5(f)-(h). One can see in the return map that the derivative of the curve for $x_{n}$ in the range from 0.5 to 1 is almost zero. Also there are regions with gradients lower and greater that 1 . This manifests itself 

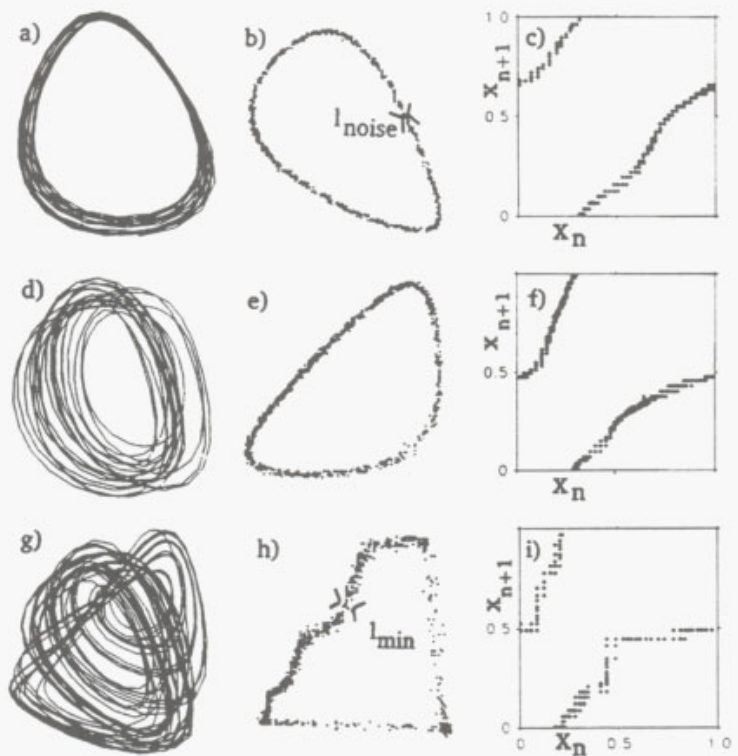

Fig. 5. Reconstructed orbits, Poincaré sections and return maps for driving frequency $f_{d}=0.700 \mathrm{MHz}$ and driving amplitudes: (a) to (c) $V_{d}=0.14 \mathrm{~V}$ well below (d) to (f) $V_{d}=0.63 \mathrm{~V}$ below and (g) to (i) $V_{d}=0.77 \mathrm{~V}$ at the critical amplitude.

in stretching and folding of the attractor and in an inhomogeneous distribution of the intersection points in the Poincare section. A reconstruction of the return map above the critical amplitude was not possible because of the immediate overlapping of mode locked resonances.
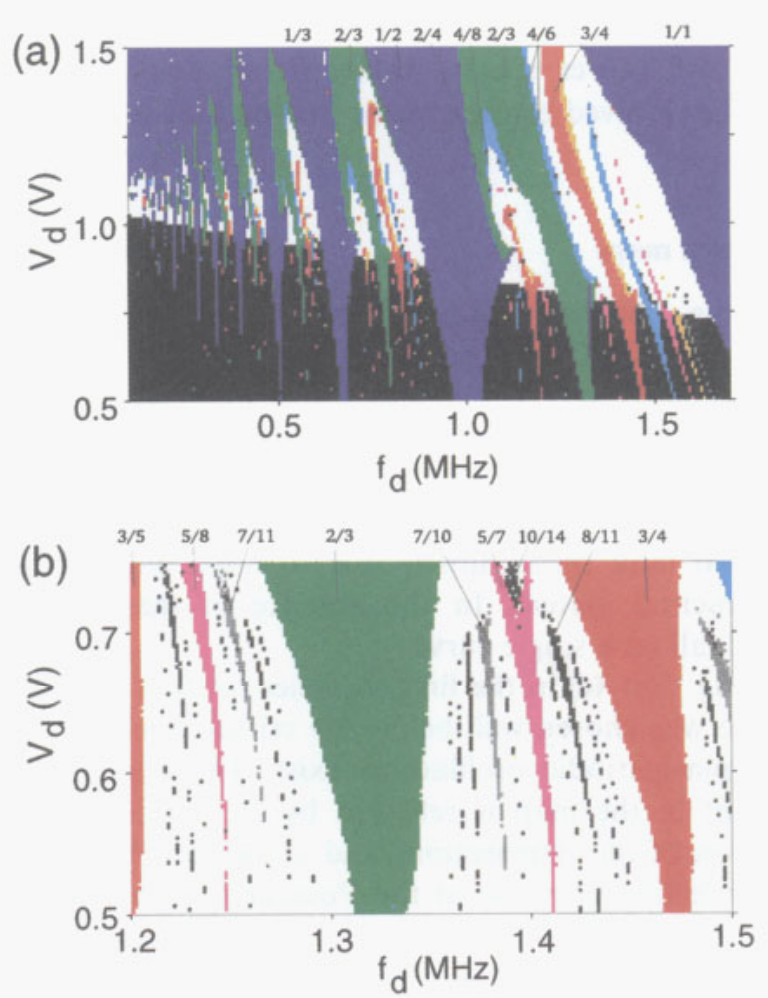

Fig. 6. Experimental phase diagrams for drive amplitude $V_{d}$ and frequency $f_{d}$. The maximum resolved numerator $\mathrm{P}$ was 14 and it was not distinguished between quaiperiodic and chaotic regimes. Coloring is as follow: Quasiperiodic regimes are black, chaotic regimes white and the mode locked regimes are: color (P): blue (1), green (2), red (3), lightblue (4), pink (5), yellow (6). The critical line in (a) (upper diagram) was plotted from the measured Devil's staircase in Fig. 7. by hand and then the quasiperiodic regime below was colored black. Diagram (b) is a part of (a) but recorded with higher resolution.

\section{Phase diagrams}

To get an overall view of the system, phase diagrams $\left(f_{d}, V_{d}\right)$ with a resolution of $200^{*} 100$ points were recorded. The numerator $P$ and denominator $Q$ were automatically determined by the computer. The maximum resolved numerator $P$ was 14 and it was not distinguished between quasiperiodic and frustrated chaotic regimes.

The phase diagrams in Fig. 6 allow the following conclusions: There are regimes in the $(K, \Omega)$ phase space where the winding number assumes rational values corresponding to Arnol'd tongues. The widths of the Arnol'd tongues grow with increasing amplitude. The sequence of the mode locking regimes follows the Farey tree. A critical line exists which divide the diagram into two sections. Below the line quasiperiodic states (black) and mode locking (colored) alternate. Above the line the Arnol'd tongues begin to overlap which indicates as chaos (white). At the right border of the tongues above the critical line period doubling occurs [for example $1 / 2$ (blue) to $2 / 4$ (green) and to $4 / 8$ (lightblue)]. Also the self similarity of the Arnol'd tongues with locking ratios $1 / Q$ can be observed. There is the same structure but with different scale for the $1 / 1,1 / 2,1 / 3,1 / 4$ etc. tongues (blue).

\section{Scaling structure of the devil's staircase}

In order to characterize the set of quasiperiodic states where the transition from quasiperiodicity to chaos takes place it is necessary to determine the critical line. The critical line is given as a lower limit for the occurrence of period doubling and the appearance of overlapping mode locked resonances. With this criteria several critical amplitudes were determined by hand and the whole critical line was calculated by linear interpolation. Now the Devil's staircase could be recorded automatically by computer with a frequency resolution of 6000 steps and distinguishing the mode locked oscillations up to a maximal numerator of $P=40$.

In the so measured Devil's staircase (Fig. 7) it was possible to resolve 312 different steps. The inset is a part of the whole staircase but plotted with higher resolution. This experimental staircase shows a very high self similarity and is almost identical to the staircase calculated from the critical circle map.

To demonstrate the self-similarity of the staircase in more detail the width $\Delta(P / Q)$ of the steps of the staircase are

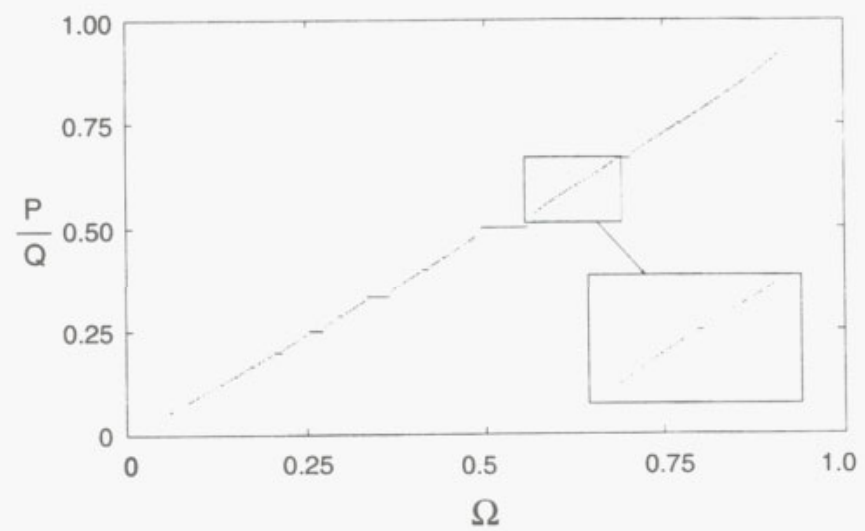

Fig. 7. Experimental Devil's staircase with 312 resolved mode locking steps. The inset shows the self-similarity. 
(a)

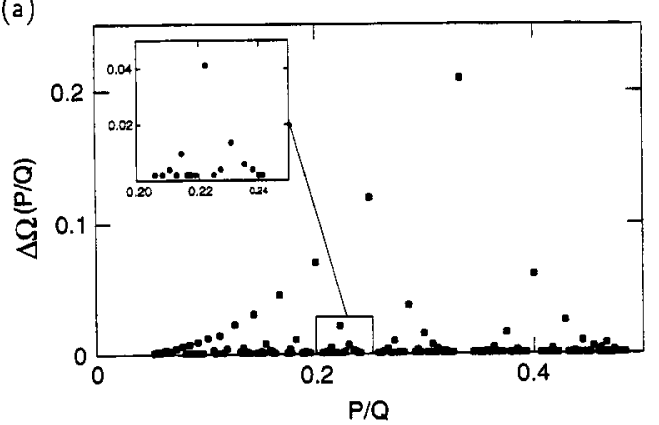

(b)

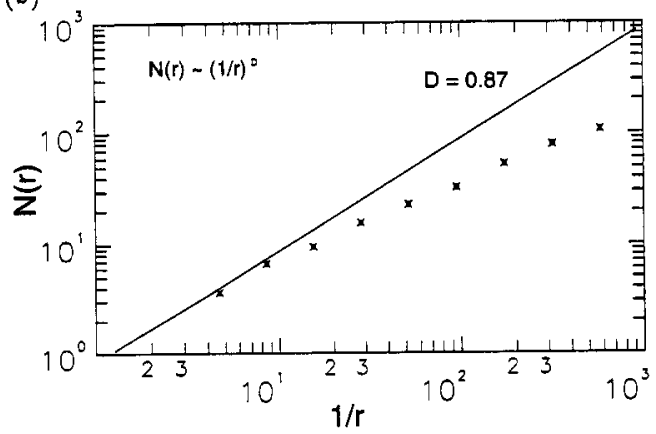

and the spatial degrees of freedom of the current filament for multifrequency oscillation were emphasized.

Driving the self-generated oscillations with an external periodic voltage quasiperiodicity, mode locking, overlap of resonances and period doubling could be observed depending on frequency and amplitude of the external force. The driven oscillations were characterized by return maps, high resolution phase diagrams and the Devil's staircase and it was shown that the staircase is self similar and complete. From this the fractal dimension of the Devil's staircase could be determined to $D=0.87$ in good agreement with the circle map theory.

These results give new experimental evidence of the universality of the transition from quasiperiodicity to chaos.

\section{Acknowledgements}

We thank E. Bauser, Max-Planck-Institut für Festkörperforschung, Stuttgart, for provision of the sample and Rohde \& Schwarz for the premature provision of a frequency synthesizer. This work was supported by the Deutsche Forschungsgemeinschaft.

Fig. 8. (a) Width of the mode locking steps versus winding number. Note the self-similarity of the diagram with the inset. (b) Plot of $\log _{10} N(r)$ vs. $\log _{10}(1 / r)$ for the critical circle map (solid line) and for the experimental Devil's staircase (points).

plotted versus $P / Q$ in Fig. 8(a). The inset in this figure shows again the self-similarity. This is also illustrated in the harmonic sequence $1 / Q$.

Besides the self-similarity the staircase should be complete. The staircase is called complete, ' when the mode locked intervals cover the entire $\Omega$ axis. This can be tested after Jensen $e t$ al. [16] by calculating the total width $S(r)$ of all steps which are larger than a given scale $r$. This yields the space between the steps $1-S(r)$ and one can get the number of holes by

$N(r)=\frac{1-S(r)}{r} \sim\left(\frac{1}{r}\right)^{D}$

which scales like $(1 / r)^{D}$. The exponent $D$ is the fractal dimension of the Cantor set of quasiperiodic intervals which is the complementary set to the mode locked intervals on the $\Omega$ axis.

In Fig. 8(b) the results from the measured staircase are plotted in a $\log -\log$ scale as points together with the results from the circle map for $D=0.87$ plotted as solid line. There is a good agreement for small $1 / r$ of the calculated points with the solid line. The discrepancy for big $1 / r$ i.e. for small quasiperiodic holes may be caused by drift in the experimental system. From this the fractal dimension of the Cantor set of the quasiperiodic states at the measured Devil's staircase can be determined giving $D=0.87$ in good agreement with the circle map theory.

\section{Summary}

The self-generated current oscillations in $n-G a A s$ were briefly described and the importance of the Lorentz force

\section{References}

1. Fein, A., Heutmaker, M. and Gollub, J., Physica Scripta T9, 79 (1985).

2. Stavans, J., Heslot, F. and Libchaber, A., Phys. Rev. Lett. 55, 596 (1985).

3. Jensen, M. H., Kadanoff, L. P., Libchaber, A., Procaccia, I. and Stavans, J., Phys. Rev. Lett. 55, 2798 (1985).

4. Cumming, A. and Linsay, P. S., Phys. Rev. Lett. 59, 1633 (1987)

5. Su, Z., Rollins, R. and Hunt, E., Phys. Rev. A36, 3515 (1987).

6. Barkley, D. and Cumming, A., Phys. Rev. Lett. 64, 327 (1990).

7. Martin, S. and Martienssen, W., Phys. Rev. Lett. 56, 1522 (1986).

8. Gwinn, E. and Westervelt, R. Phys. Rev. Lett. 57, 1060 (1986).

9. Held, G. and Jeffries, C., Phys. Rev. Lett. 56, 1183 (1986).

10. Teitsworth, S. and Westervelt, R., Phys. Rev. Lett. 56, 516 (1986).

11. Kim, Y. Phys. Rev. A39, 4801 (1989).

12. Feigenbaum, M. Kananoff, L. and Shenker, S., Physica DS, 370 (1982).

13. Shenker, S., Physica D5, 405 (1982).

14. Rand, D., Ostlund, S., Sethna, J. and Siggia, E., Phys. Rev. Lett. 49, 132 (1982).

15. Ostlund, S., Rand, D., Sethna, J. and Siggia, E., Physica D8, 303 (1983).

16. Jensen, M. H., Bak, P. and Bohr, T., Phys. Rev. A30, 1960 (1984).

17. Jensen, P. C. M. and Kandanoff, L., Phys. Rev. Lett. 55, 343 (1985).

18. Halsey, T. C., Jensen, M. H., Kadanoff, L. P., Procaccia, I and Shraiman, B. I., Phys. Rev. A33, 1141 (1986).

19. Aoki, K., Kobayashi, T. and Yamamoto, K., J. Phys. Soc. Jpn. 51, $2373(1982)$

20. Teitsworth, S., Westervelt, R. and Haller, E., Phys. Rev. Lett. 51, 825 (1983).

21. Held, G., Jeffries, C. and Haller, E., Phys. Rev. Lett. 52, 1037 (1984).

22. Peinke, J., Mühlbach, A., Huebener, R. and Parisi, J., Phys. Lett. A108, 407 (1985).

23. Brandl, A., Geisel, T. and Prettl, W., Europhys. Lett. 3, 401 (1987).

24. Spangler, J., Brandl, A. and Prettl, W., Appl. Phys. A48, 143 (1989).

25. Schöll, E., "Nonequilibrium Phase Transitions in Semiconductors" (Springer Verlag, Berlin 1987).

26. Spangler, J., Margull, U. and Prettl, W., Phys. Rev. B45, 12137 (1992)

27. Hüpper, G. and Schöll, E., Phys. Rev. Lett. 66, 2372 (1991).

28. Grassberger, P. and Procaccia, I., Phys. Rev. Lett 50, 346 (1983)

29. Newhouse, S., Ruelle, D. and Takens, F., Comm. Math. Phys. 64, 35 (1978).

30. Feigenbaum, M., J. Stat. 19, 25 (1978). 